\title{
Prefrontal Brain Activation in Subtypes of Attention Deficit Hyperactivity Disorder: A Functional Near-Infrared Spectroscopy Study
}

\author{
Miray Altınkaynak' (D), Ayşegül Güven' ${ }^{1}$ (D), Nazan Dolu² (D), Meltem İzzetoğlu³ (D), Esra Demirci ${ }^{4}$ (D), \\ Sevgi Özmen ${ }^{4}$ iD, Ferhat Pektaş ${ }^{5}$ (D)
}

\author{
Department of Biomedical Engineering, Erciyes University School of Engineering, Kayseri,Turkey \\ 2Department of Physiology, Başkent University School of Medicine, Ankara, Turkey \\ 32Department of Electrical and Computer Engineering, Villanova University School of Engineering, Villanova, USA \\ ${ }^{4}$ Department of Child and Adolescent Psychiatry, Erciyes University School of Medicine, Kayseri, Turkey \\ 5Department of Physiology, Altınbaş University School of Medicine, İstanbul, Turkey
}

Cite this article as: M. Altınkaynak, A. Güven, N. Dolu, M. İzzetoğlu, E. Demirci, s. Özmen, F. Bektaş, "Prefrontal Brain Activation in Subtypes of Attention Deficit Hyperactivity Disorder: A Functional Near-Infrared Spectroscopy Study", Electrica, vol. 18, no: 2, pp. 256-262, 2018.

\begin{abstract}
According to clinical symptoms, attention deficit and hyperactivity disorder (ADHD) is categorized into three groups: the predominantly inattentive subtype (ADHD-I), the predominantly hyperactive-impulsive subtype (ADHD-HI), and the combined subtype (ADHD-C). Recent advances in neuroimaging have demonstrated new approaches for assessing the ADHD subtypes with underlying pathophysiology. This study aims to examine the hemodynamic response and reaction time (RT) in healthy children and the ADHD subtypes as measured by functional near-infrared spectroscopy (fNIRS) during an auditory oddball attention task. The sample was made up of 40 children divided into four groups: control group ( $n=14)$, ADHD-I group ( $n=9$ ), ADHD-HI group ( $n=6)$, and ADHD-C group ( $n=11)$. The target responses were identified and were grand-averaged for each participant. Right prefrontal cortex hemodynamic responses and groups performances on RT were compared between subtypes and between controls and subtypes. Functional nearinfrared spectroscopy indicated that while control subjects exhibited higher activation than all ADHD subtypes, the ADHD subtypes did not differ from one another. Relative to control subjects, a longer RT was observed in all ADHD subtypes. The ADHD-I group showed significantly longer RTs compared to the ADHD-HI and ADHD-C groups. This study can bring a new perspective to the continuing controversy about ADHD subtypes, and the findings may help in the evaluation of fNIRS, RT, and RT variability studies in ADHD.
\end{abstract}

Keywords: ADHD subtype, functional near-infrared spectroscopy, reaction time, oxyhemoglobin

\section{Corresponding Author:}

Miray Altınkaynak

E-mail:

eem.miray@gmail.com

Received: 28.02 .2018

Accepted: 19.04 .2018

(c) Copyright 2018 by Electrica

Available online at

http://electrica.istanbul.edu.tr

DOI: 10.26650/electrica.2018.99730

\section{Introduction}

Attention Deficit Hyperactivity Disorder (ADHD) is a common, heterogeneous, neurodevelopmental disorder in school age children, which is characterized by inattention, hyperactivity and impulsivity symptoms [1]. The clinical symptoms of ADHD are categorized as persistent inattention (ADHD-I), hyperactivity-impulsivity (ADHD-H) and a combination of both (ADHD-C) according to DSM-IV or DSM-V criteria [2].

Recent neuroimaging studies have demonstrated new approaches for assessment of the ADHDs' brain functions. While some of these studies indicated structural abnormalities, some other studies illustrated functionally reduced activity in the frontal lobe of patients suffering from ADHD [3-6]. However, these studies often disregarded the differences between subtypes. Differences of brain structure and functions between ADHD subtypes were investigated in a few neuroimaging studies. The existing, limited literature is inconsistent; some of these studies did not detect differences on neuroimaging measures while some showed significant differences between subtypes. Saad et al. [7] investigated global brain network measures and grey matter volume of ADHD subtypes and healthy controls by Magnetic resonance imaging (MRI) and found clinical distinction between the ADHD-I and ADHD-C subtypes. Vilgis [8] used also MRI to examine brain volume differences between ADHD subtypes and found no significant 
differences. A task-based functional MRI (fMRI) study indicated more dysfunctions in ADHD-I compared to the ADHD-C subtype [9]. Seidman et al. [10] reviewed heterogeneity of executive functions among subtypes of ADHD and reported that there were more similarities than differences. Barkley [11] found differences between subtypes of ADHD in terms of executive functions, memory, and focused attention. In this current study we investigated neuroimaging profiles of ADHD subtypes with optical brain imaging using functional near-infrared spectroscopy (fNIRS). fNIRS measures the event related hemodynamic response of the brain with concentration changes of oxygenated and deoxygenated hemoglobin. fNIRS provides advantages due to its safety, sensitivity, cost-effectiveness and tolerance to body motion, thus is considered suitable for the clinical evaluations of ADHD [12]. Various fNIRS studies have sought to assess the hemodynamic changes of ADHD patients. These studies are particularly focused on prefrontal cortex (PFC) activity and consistently showed neural dysfunctions in children with ADHD compared to healthy controls [13-15]. In this study we investigated ADHDs' frontal brain functions during an auditory stimulation with hemodynamic changes, specifically in oxy- $\mathrm{Hb}$.

Another more consistent finding in ADHD children is that they have slower and more variable reaction times (RTs) on a number of different tasks compared to healthy controls [16]. RT is the length of time between the presentation of the stimulus and the initiation of the subject's response. It is an important and informative tool in the study of cognitive ability in psychology and other disciplines. Studies have demonstrated that increased RT variability in ADHD is mostly related to extremely slow responses that are caused by periodic lapses of attention in goal-directed processing [17]. The literature is inconsistent with respect to differences between ADHD subtypes in terms of inhibitory control, where some of these studies showed the ADHD-C type had longer RTs and increased commission errors, some showed the ADHD-I subtype had slower processing speed compared to other subtypes and some others found no differences among subtypes in terms of RT and task errors [18]. Thus more work is also needed to evaluate differences in the RT of ADHD subtypes.

Because the results of neurocognitive studies are unclear about whether the ADHD subtypes are variants of the same condition (as current clinical view) or completely different disorders, in this study, we used fNIRS to provide new insights into the evalution of distinction among the subtypes in terms of oxygenation of PFC and RT to target trials during an auditory oddball attention task.

\section{Material and Methods}

\section{Participants}

The subjects were comprised of 40 children that were classified into four groups: control group with 14 participants ( mean $=10.33$; SD $=2.12$; range 7-12 years); ADHD-I group with 9 participants (mean age $=9.66 \pm 2.95$; range $7-12$ years);
ADHD-HI group with 6 participants 8.16 (mean age: 8.16 \pm 0.75 ; range $7-12$ years); and ADHD-C group with 11 participants 8.16 (mean $=10 \pm 1.61$; range $7-12$ years). ADHD subtypes were carefully identified by two experienced pediatric psychiatrists according to the DSM IV diagnostic criteria. All ADHD participants were medication naïve. Controls were included in the study after a standard clinical assessment including neurological, endocrine and psychiatric evaluations. Participants were all right handed, Turkish and had normal hearing functions according to the Rinne and Weber test. According to The Wechsler Intelligence Scale for Children-Revised (WISC-R), IQ scores of all participants were over 80 [19]. The study was approved by the local ethics committee of Erciyes University and was in accordance with the Declaration of Helsinki. All participants assent and parental permission were obtained.

\section{Task}

fNIRS recordings were obtained while participants performed the auditory 'oddball' paradigm. In this study 160 auditory stimuli that contain standard $(2000 \mathrm{~Hz})$ and target $(1500 \mathrm{~Hz})$ trials were presented in a random order. The target trials comprised $20 \%$ of the whole stimuli. The interstimulus intervals were randomized between 1250 and 2500 msec. Prior to the first run of the experiment, the participants were asked to respond to target trials by pressing the button. During the experiment the participants instructed not to move, speak or blink too much in order to avoid noises and stabilize the blood flow in the fNIRS channels.

\section{fNIRS Data Acquisition and Pre-processing}

In this study, we used a 16 channel $(\mathrm{CH})$ continuous wave fNIRS system that has four light sources at two different wavelengths with $730 \mathrm{~nm}$ and $850 \mathrm{~nm}$ and ten photo detectors separated from the sources by $2.5 \mathrm{~cm}$. The sampling frequency was $2 \mathrm{~Hz}$. Hemodynamic changes were calculated using the modified Beer-Lambert law [20]. 16 channel fNIRS signal belongs to a participant in the control group presented in Figure 1. First, the raw fNIRS intensity measurements were low-pass filtered with a cut-off set to $0.14 \mathrm{~Hz}$ [21] to remove noises derived from movement artifacts, heart pulsation and respiration. Then the data of each channel were averaged across 32 target responses for each subject. Target responses identified $3 \mathrm{~s}$ before the target stimuli period onset to $10 \mathrm{~s}$ after the target stimuli (Figure 2). RT of participants to correctly identified target trials were detected with Matlab R2015a automatically by calculating the length of time between the presentation of the target stimulus and the initiation of the subject's response. Mean RT (MRT) for each participant was computed by averaging RTs.

\section{Region of Interest}

Inconsistent with previous studies (see results) the analyses of fNIRS revealed that compared to healthy controls, ADHD children (in all subtypes), showed reduced increases in the concentration of oxy- $\mathrm{Hb}$ particularly for channels located in the right PFC. So, 
we defined this region as region of interest $(\mathrm{ROI})$ and focused on these channels (CHs 9,10,11,12,13,14,15,16) (Figure 3). We calculated the average of the integral value of oxy-Hb for the selected region of interest $(\mathrm{ROI})$ for each participant.

\section{Statistical Analyses}

We compared the variables between the ADHD subtypes and

Table 1. Values of variables for each group (mean \pm standart deviation)

\begin{tabular}{lcccc}
\hline & Control & ADHD-I & ADHD-HI & ADHD-C \\
\hline Oxy-Hb & $1.87 \pm 0.72$ & $1.02 \pm 0.5$ & $1.021 \pm 0.65$ & $1.21 \pm 0.51$ \\
\hline RT(ms) & $513 \pm 77.7$ & $1046 \pm 379$ & $728.4 \pm 376.5$ & $717 \pm 225$ \\
\hline
\end{tabular}

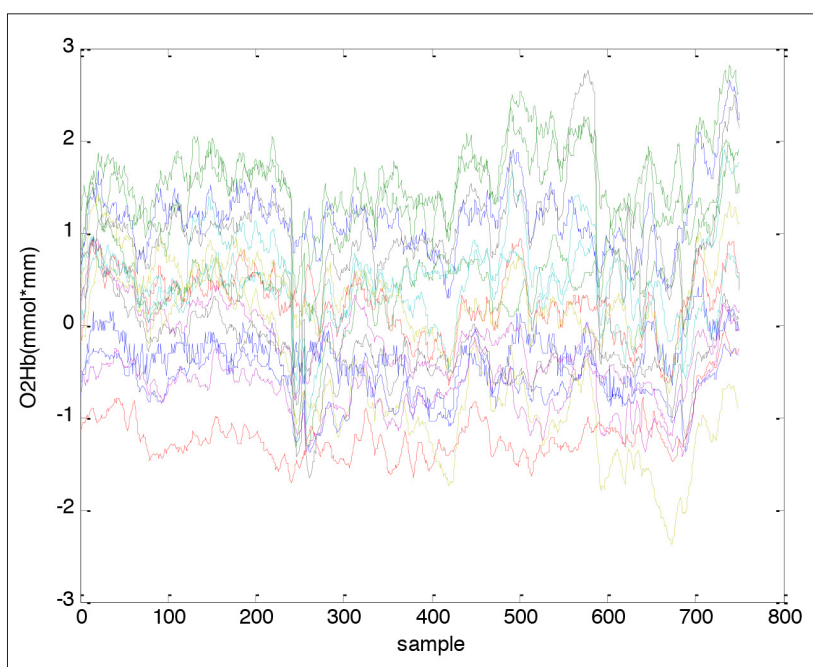

Figure 1. 16 channel raw fNIRS signal belongs to a participant in the control group the control group using a student's t-test. Shapiro-Wilk normality test was used to confirm the normal distribution of variables. The statistical threshold was chosen as 0.05. Statistical analyses were performed using the SPSS (Statistical Package for Social Sciences) for Windows Version 16.0 (SPSS Inc.; Chicago IL, USA) software.

\section{Results}

Grand average of integrated oxy-Hb for all target responses belonging to one participant of each group are illustrated in Figure 4. fNIRS indicated that while control subjects exhibited higher activation than all ADHD subtypes, ADHD subtypes did not differ statistically from one another. All ADHD subtypes showed longer RT $(p<0.01)$ than controls. ADHD-I group showed significantly longer RTs compared to ADHD-HI and ADHD-C group. Statistical analysis results are presented in Table 1-3, separately.

\section{Conclusion}

The present study aimed to investigate whether ADHD subtypes and healthy controls differ in terms of RT and oxygenation of PFC during an auditory oddball attention task. Our fNIRS results were inconsistent with previous studies revealed that ADHD children (in all subtypes) showed reduced activation for channels located in the right PFC compared to healthy controls $[13,14]$. Although the ADHD-I group had lower oxy-Hb values compared to the other subtypes the differences were statistically non-significant (Figure 4). ADHD subtypes did not differ from one another in terms of concentration of oxy- $\mathrm{Hb}$. This finding confirms that inattentive symptomatology is common to all subtypes of ADHD [22]. In RT results; relative to control subjects, a longer RT was observed in all ADHD subtypes. The ADHD-I group showed significantly longer RTs compared to the ADHD-HI and ADHD-C groups. The results are consistent with previous studies $[11,16]$. Barkley indicated that ADHD-I






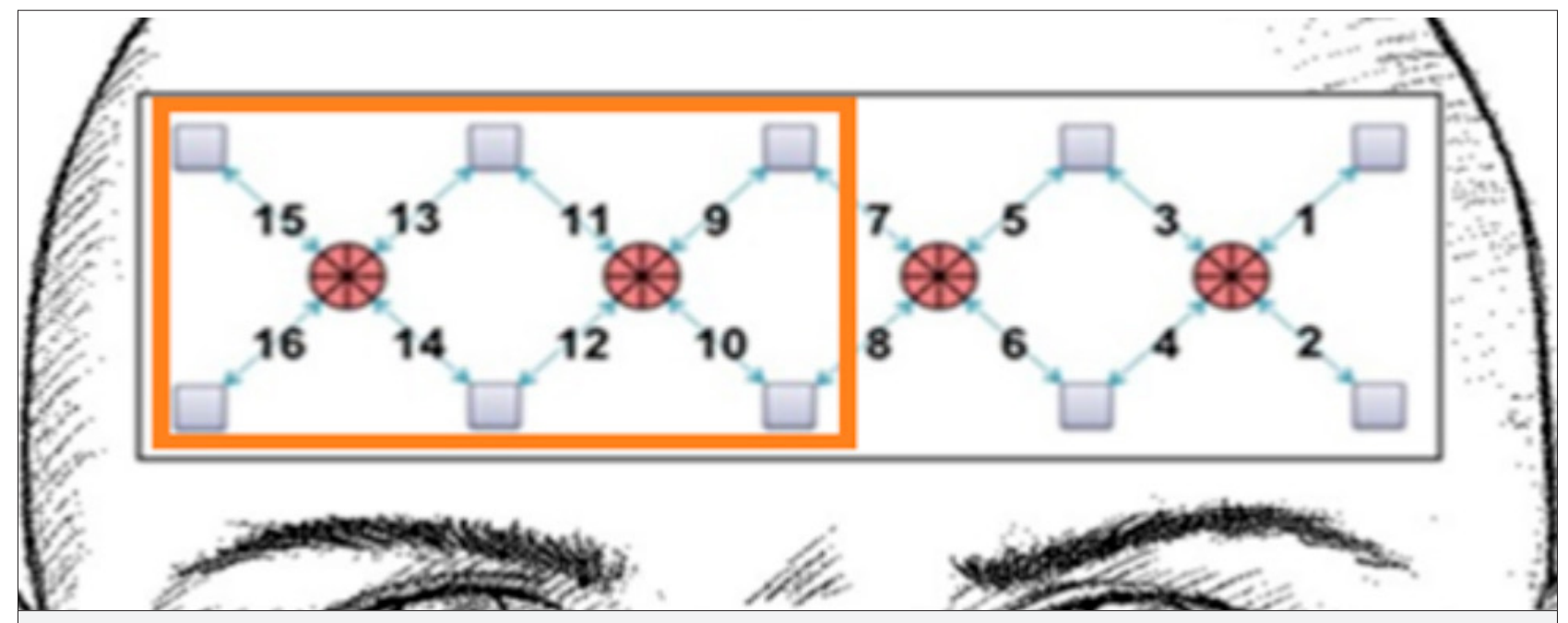

Figure 3. Specified $\mathrm{ROI}$

Table 2. Comparison between controls and ADHD subtypes

\begin{tabular}{lcccccc}
\hline & \multicolumn{2}{c}{$\begin{array}{c}\text { Control vs. } \\
\text { ADHD-I }\end{array}$} & \multicolumn{2}{c}{$\begin{array}{c}\text { Control vs. } \\
\text { ADHD-HI }\end{array}$} & \multicolumn{2}{c}{$\begin{array}{c}\text { Control vs. } \\
\text { ADHD-C }\end{array}$} \\
\cline { 2 - 7 } & $\mathbf{t}$ & $\mathbf{p}$ & $\mathbf{t}$ & $\mathbf{p}$ & $\mathbf{t}$ & $\mathbf{p}$ \\
\hline Oxy-Hb & 3.36 & $0.003^{*}$ & 2.4 & $0.025^{*}$ & 2.57 & $0.017^{*}$ \\
\hline RT & 5.34 & $0.000^{*}$ & 2.18 & $0.042^{*}$ & 3.25 & $0.003^{*}$ \\
\hline
\end{tabular}

Table 3. Comparison between ADHD subtypes

\begin{tabular}{lcccccc}
\hline & $\begin{array}{c}\text { ADHD-I vs } \\
\text { ADHD-C }\end{array}$ & \multicolumn{2}{c}{$\begin{array}{c}\text { ADHD-I vs } \\
\text { ADHD-HI }\end{array}$} & \multicolumn{2}{c}{$\begin{array}{c}\text { ADHD-C vs } \\
\text { ADHD-HI }\end{array}$} \\
\hline t & $\mathbf{p}$ & $\mathbf{t}$ & $\mathbf{p}$ & $\mathbf{t}$ & $\mathbf{p}$ \\
\hline RT & 0.96 & 0.348 & 0.007 & 0.994 & 0.72 & 0.486 \\
\hline
\end{tabular}

*: significant

reflected impaired speed of processing compared to ADHD-C [11]. Our results in RT propose it will be better to distinguish ADHD subtypes in RT and RT variability studies to obtain more reliable results.

Recently, right prefrontal activation served as an objective biomarker for fNIRS measurement in ADHD patients in many studies [13-15]. So we focused on this region. In many studies it has been shown that oxy- $\mathrm{Hb}$ is more sensitive and reliable than deoxy- $\mathrm{Hb}$ and total heamoglobin for the assessment of cortical activation during a cognitive task [23, 24] The oxy$\mathrm{Hb}$ has been identified as a discriminatory feature for ADHD and controls in many previous fNIRS studies [13-15]. We selected an easy, short task containing a standard and target paradigm with random squence that can be appropriate for ADHD children. This current study was one of a few studies using the selective auditory attention task to investigate the fNIRS in ADHD children.

Neuroimaging and neuropsychological studies on distinctiveness of the ADHD subtypes are inconsistent and unclear and more study is needed [25]. Recently this topic has been studied often with different neuroimaging methods including, MRI, fMRI, Diffusion Tensor imaging (DTI) by examining structural and functional differences among groups. Recently Al Amin et al. [26] found more reduction in hippocampal volumes in the ADHD-C compared to the ADHD-I group. Geurts et al. [27] investigated executive and non-executive functioning in ADHD subtypes and found deficits in all subtypes that do not differ from another. Ercan et al. [28] examined microstructural properties of white matter in ADHD subtypes with DTI and found no significant difference among ADHD subtypes. Barkley [11] found differences among subtypes of ADHD in terms of executive functions, memory, and attention and hypothesized that these subtypes are completely different disorders. Similarly, Dobson-Patterson et al. [29] found statistically significant differences among ADHD subtypes in attention, memory and executive functions. Seidman et al. [10] reviewed heterogeneity of executive functions among subtypes of ADHD and reported that there were more similarities than differences. Solanto et al. [30] observed no significant group differences among the subgroups of ADHD in task (go/no-go paradigm) performance in fMRI. Similarly Altınkaynak et al. [31] found no differences in hemodynamic measures of subtypes of ADHD during a cognitive task. As seen in the above mentioned literature the results of neurocognitive studies are inconsistent and there is a continuing controversy of whether ADHD subtypes are different disorders or as in the current clinical view, they are variants of the same condition. We investigated this problem with the recently popular imaging method: fNIRS. In summary, our results show ADHD subtypes did not differ significantly from another in prefrontal cortex oxygenation during an executive task. So we suggest the subtypes are variants of the same condition. 


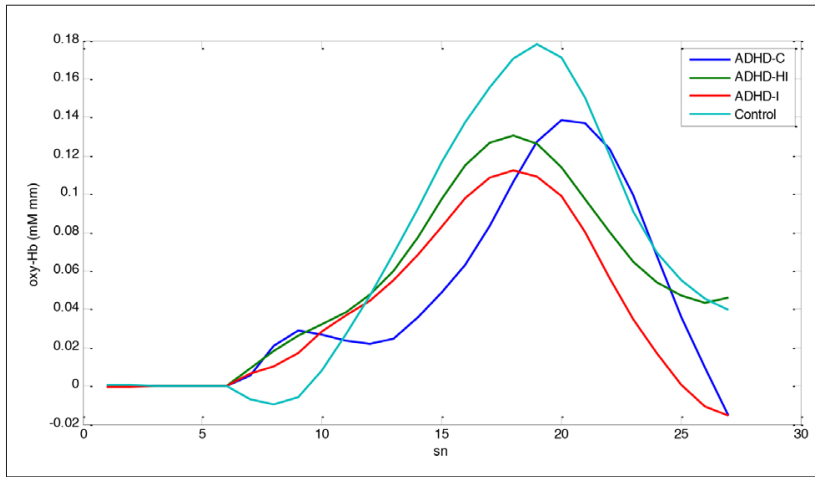

Figure 4. The average of target responses of oxy- $\mathrm{Hb}$ signal of ADHD subtypes and control in the right PFC

The study will bring a new perspective to the continuing controversy about ADHD subtypes and the findings may help in the reliable evaluation of fNIRS, RT and RT variability studies in ADHD.

A limitation of the present study was the relatively small sample size. Anyway, the ADHD-HI group was less compared to the others. This is because the ADHD-Hl subtype diagnosis is far less common and generally ADHD subtype studies are conducted on the ADHD-C and ADHD-I groups. Notwithstanding the lower population of the group, we included the ADHD-HI group in the study in order to evaluate all three subtypes of ADHD.

Ethics Committee Approval: Ethics committee approval was received for this study from Erciyes University.

Informed Consent: Informed consent was obtained from the patients who participated in this study.

Peer-review: Externally peer-reviewed.

Conflict of Interest: The Authors have no conflicts of interest to declare.

Financial Disclosure: This study was supported by the TUBITAK under project number 114 S47.

\section{References}

1. J. Biederman, S.V. Faraone, "Attention-deficit hyperactivity disorder", Lancet, vol. 366, no. 9481, pp. 237-248, 2005.

2. American Psychiatric Association, "Diagnostic and Statistical Manual of Mental Disorders", $4^{\text {th }}$ edition, 2000.

3. K. S. Rosch, D. Crocetti, K. Hirabayashi, M. B. Denckla, S. H. Mostofsky, E. M. Mahone, "Reduced subcortical volumes among preschool-age girls and boys with ADHD", Psychiatry Res Neuroimaging, vol. 271, pp. 67-74, 2018.

4. C. Sridhar, S. Bhat, U. R. Acharya, H. Adeli, G. M. Bairy, "Diagnosis of attention deficit hyperactivity disorder using imaging and signal processing techniques", Comput Biol Med, vol. 88, pp. 93-99, 2017.

5. Y. Monden, H. Dan, M. Nagashima, I. Dan, D. Tsuzuki, Y. Kyutoku, Y. Gunji, T. Yamagata, E. Watanabe, M.Y. Momoi, "Right prefrontal activation as a neuro-functional biomarker for monitoring acute effects of methylphenidate in ADHD children: An fNIRS study", Neuroimage Clin, vol. 1, no.1, pp. 131-140, 2012.

6. G. Bush, E. M. Valera, L. J. Seidman, "Functional neuroimaging of attention-deficit/hyperactivity disorder: A reviewand suggested future directions", Biol Psychiatry, vol. 57, no. 11, pp. 1273-1284, 2005.

7. J. F. Saad, K.R. Griffiths, M. R. Kohn, S. Clarke., L. M. Williams, M. S. Korgaonkar, "Regional brain network organization distinguishes the combined and inattentive subtypes of Attention Deficit Hyperactivity Disorder", Neuroimage Clin, vol. 22, no.15, pp. 383-390, 2017.

8. V. Vilgis, L. Sun, J. Chen, T. J. Silk, A. Vance, "Global and local grey matter reductions in boys with ADHD combined type and ADHD inattentive type", Psychiatry Res Neuroimaging, vol. 254, pp. 119126, 2016.

9. A. J. Orinstein, M. C. Stevens, "Brain activity in predominantly-inattentive subtype attention-deficit/hyperactivity disorder during an auditory oddball attention task", Psychiatry Res. Neuroimaging, vol. 223, no. 2, pp. 121-128.2014.

10. L. J. Seidman, "Neuropsychological functioning in people with ADHD across the lifespan", Clin Psychol Rev, vol. 26, no. 4, pp.466485, 2006.

11. R. A. Barkley, "Behavioral inhibition, sustained attention, and executive functions: constructing a unifying theory of ADHD", Psychol Bull, vol. 121, no. 1, pp.65-94, 1997.

12. A. C. Ehlis, S. Schneidera, T. Dresler, A. J. Fallgatter, "Application of functional near-infrared spectroscopy in psychiatry", Neurolmage, vol. 85, no. 1, pp. 478-488, 2014.

13. Y. Monden, I. Dan, M. Nagashima, H. Dan, M. Uga, T. Ikeda, D. Tsuzuki, Y. Kyutoku, Y. Gunji, D. Hirano, T. Taniguchi, H. Shimoizumi, E. Watanabe, T. Yamagata, "Individual classification of ADHD children by right prefrontal hemodynamic responses during a go/no-go task as assessed by fNIRS", Neuroimage Clin, vol.9, pp.1-12, 2015.

14. S. Jourdan Moser, S. Cutini, P. Weber, M. L. Schroeter, "Right prefrontal brain activation due to Stroop interference is altered in attention-deficit hyperactivitydisorder-A functional near-infrared spectroscopy study" Psychiatry Res, vol. 173, no. 3, pp. 190-19, 2009.

15. Y. Monden, H. Dan, M. Nagashima, I. Dan, D. Tsuzuki, Y. Kyutoku, Y. Gunji, T. Yamagata, E. Watanabe, M. Y. Momoi, "Right prefrontal activation as a neuro-functional biomarker for monitoring acute effects of methylphenidate in ADHD children: An fNIRS study" Neurolmage Clin, vol. 1, no. 1, pp. 131-140, 2012.

16. D. R. Coghill, S. Seth, S. Pedroso, T. Usala, J. Currie, A Gagliano, "Effects of Methylphenidate on Cognitive Functions in Children and Adolescents with Attention-Deficit/Hyperactivity Disorder: Evidence from a Systematic Review and a Meta-Analysis", Biol Psychiatry, vol. 76, no. 8, pp. 603-15, 2014.

17. L. Tamm, M. E. Narad, T. N. Antonini, K. M. O'Brien, L. W. Hawk, J. N. Epstein, "Reaction time variability in ADHD: A review", Neurotherapeutics, vol. 9, no. 3, pp. 500-508, 2012.

18. M. V. Solanto, S. N. Gilbert, A. Raj, J. Zhu, S. Pope-Boyd, B. Stepak, L. Vail, J. H. Newcorn "Neurocognitive functioning in AD/HD, Predominantly Inattentive Subtype", J Abnorm Child Psychol, vol. 35, no. 5, pp.729-744, 2007.

19. D. Wechsler, "WISC-R Manuel for the Wechsler Intelligence Scale for Children Revised", New York: Psychological Corporation, 1972.

20. M. Cope, D. T. Delpy, "System for long-term measurement of cerebral blood flow and tissue oxygenation on newborn infants by infrared transillumination", Med Biol Eng Comput, vol. 26, no. 3, pp.289-294, 1988.

21. M. Izzetoglu, K. Izzetoglu, S. Bunce, H. Ayaz, A. Devaraj, B. Onaral, K. Pourrezaei, "Functional near-infrared neuroimaging". IEEE Trans Neural Syst Rehabil Eng, vol. 13, no. 2, pp.153-9, 2005. 
22. E. G. Willcutt, J. T. Nigg, B. F. Pennington, M. V. Solanto, L. A. Rohde, R. Tannock, S. K. Loo, C. L. Carlson, K. McBurnett, B. B. Lahey, “Validity of DSM-IV attention-deficit/hyperactivity disorder symptom dimensions and subtypes", J Abnorm Psychol, vol. 121, no. 4, pp. 991-1010, 2012.

23. A. C. Ehlis, C. G. Bahne, C. P. Jacob, M. J. Herrmann, A. J Fallgatter, "Reduced lateral prefrontal activation in adult patients with attention-deficit/hyperactivity disorder (ADHD) during a working memory task: a functional near-infrared spectroscopy (fNIRS) study", J Psychiatry Res, vol. 42, NO. 13, pp. 1060-1067, 2008.

24. Y. Hoshi, "Functional near-infrared optical imaging: utility and limitations in human brain mapping", Psychophysiology, vol. 40, no. 4, pp. 511-520, 2003.

25. D. A. Fair, J. T. Nigg, S. Iyer, D. Bathula, K. L. Mills, N. U. Dosenbach, B. L. Schlaggar, M. Mennes, D. Gutman, S. Bangaru, J. K. Buitelaar, D. P. Dickstein, A. Di Martino; D.N. Kennedy, C. Kelly, B. Luna, J. B. Schweitzer, K. Velanova, Y. F. Wang YF, S. Mostofsky, F. X. Castellanos, M. P. Milham, “Distinct neural signatures detected for ADHD subtypes after controlling for micro-movements in resting state functional connectivity MRI data", Front Syst Neurosci, vol. 6, no. 80, pp. 1-31, 2012.
26. M. Al-Amin, A. Zinchenko, T. Geyer, "Hippocampal subfield volume changes in subtypes of Attention Deficit Hyperactivity Disorder", Brain Res, vol. 1685, pp. 1-8, 2018.

27. H. M. Geurts, S. Verté, J. Oosterlaan, H. Roeyers, J. A. Sergeant, "ADHD subtypes: do they differ in their executive functioning profile?" Arch Clin Neuropsychol, vol.20, no.4, pp. 457-77, 2005.

28. E. S. Ercan, S. Suren, A. Bacanlı, K. U. Yazıcı, C. Callı, U. A. Ardic, D. Aygunes, B. Kosova, O. Ozyurt, C. Aydın, L. A. Rohde, "Altered structural connectivity is related to attention deficit/hyperactivity subtypes: A DTI study". Psychiatry Res Neuroimaging, vol.30, no. 256, pp. 57-64, 2016.

29. R. Dobson-Patterson, J. G. O'Gorman, R. C. Chan, D. H. Shum, "ADHD subtypes and neuropsychological performance in an adult sample" Res Dev Disabil, vol. 55, pp. 55-63, 2016.

30. M. V. Solanto, K. P. Schulz, J. Fan, C. Y. Tang, J. H. Newcorn, "Event-related fMRI of inhibitory control in the Predominantly Inattentive and Combined Subtypes of AD/HD", J Neuroimaging, vol. 19, no. 3, pp. 205-212, 2009.

31. M. Altınkaynak, A. Guven, N. Dolu, M. İzzetoğlu, E. Demirci, S. Ozmen, F. Pektaş" Investigating prefrontal hemodynamic responses in ADHD subtypes: A fNIRS study", $10^{\text {th }}$ International Conference on Electrical and Electronics Engineering (ELECO), 2017, pp. 611-614. 


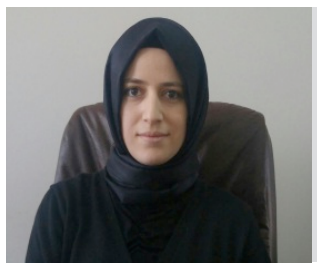

Miray Altınkaynak recieved her BSc degree from Atatürk University Electrical and Electronics Engineering and Mechanical Engineering in 2009 and 2010 respectively. She received the MSc. degree in Biomedical Engineering from Erciyes University, in 2013. She is currently a PhD student and research assistant at Biomedical Engineering Department, Erciyes University. Her research interests include biomedical signal analysis, biomedical optics and machine learning.

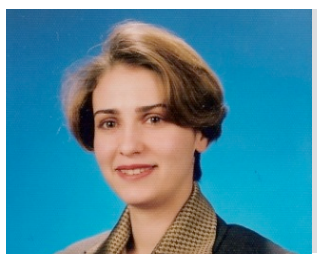

Ayşegül GÜVEN received respectively her BSc, MSc and PhD degrees from Erciyes University Electrics and Electronics Engineering in 1996, 1999 and 2006. She is currently an associate professor at the Biomedical Engineering Department of Erciyes University. Her current research interests include biomedical signal processing, medical imaging, classification and machine learning.

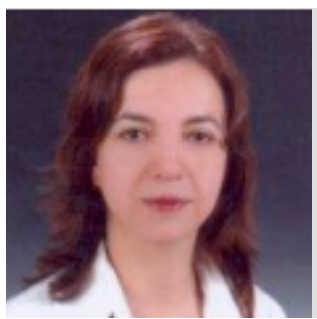

Nazan Dolu received her medical training from İstanbul University Cerrahpaşa School of Medicine, in 1989 and completed her post-doctorate research in Psychology at the Erciyes University School of Medicine, Kayseri in 1996. She worked at the Erciyes University from 1993 to 2016. Then she retired from Department of Psychology, Erciyes University Scool of Medicine in 2015. She is currently a professor at Department of Psychology, Başkent University School of Medicine. Her specific teaching and research interests are neuroscience, electrophysiology, neurophysiology and molecular biology. She authored or co-authored in 145 papers published in scientific journals and conference proceedings combined.



Meltem İzzetoğlu received the B.S. and the M.S. degrees both in Electrical and Electronics Engineering from Middle East Technical University, Ankara, Turkey, in 1992 and 1995, respectively. She received the Ph.D. degree in Electrical and Computer Engineering from Drexel University, Philadelphia, Pennsylvania, in 2002. She was a research faculty at School of Biomedical Engineering, Science and Health Systems, Drexel University, Philadelphia, PA between 2002 and 2017 where she has been an active member of Cognitive Neuroengineering and Quantitative Experimental Research (Conquer) Collaborative. Dr. Izzetoglu is currently a research professor at Electrical and Computer Engineering Department, Villanova University, Villanova, PA. Her research interests include biomedical signal analysis, neuroimaging and cognitive neuroscience, adaptive and optimal signal processing, biomedical optics, noise removal and information extraction in functional near infrared spectroscopy applications.

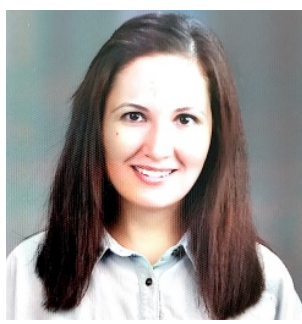

Esra DEMIRCi received her medical training from Uludag University, Faculty of Medicine, Bursa in 2006 and completed her post-doctorate research in Child and Adolescent Psychiatry at the Erciyes University School of Medicine, Kayseri in 2013. She has been working at the Department of Child and Adolescent Psychiatry, Erciyes University School of Medicine, from 2013. Her specific teaching and research interests are child and adolescent mental health, neurodevelopmental disorders especially ADHD, neuroscience, neuroimaging and also child abuse. She authored or co-authored in 97 papers published in scientific journals and conference proceedings combined.

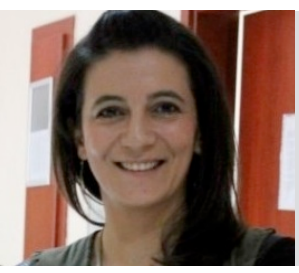

Sevgi Özmen received her medical training from Erciyes University School of Medicine, Kayseri in 2006 and completed her post-doctorate research in Child and Adolescent Psychiatry at the Erciyes University, Faculty of Medicine, Kayseri in 2012. She has been working at the Department of Child and Adolescent Psychiatry, Erciyes University School of Medicine, from 2013. Her specific teaching and research interests are child and adolescent mental health, neurodevelopmental disorders especially ADHD, neuroscience and neuroimaging.

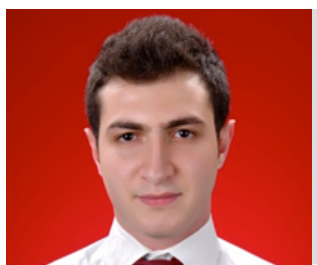

Ferhat Pektaş recieved the BSc degree, from department of biology, İnönü University in 2012, Turkey. He received the MSc. degree in Physiology department of Health Science institute from Erciyes University, in 2016. He is currently a PhD student at Department of PhysiologyErciyes University School of Medicine and research assistant at Department of Physiology, Altınbaş University School of Medicine. His research interests include neurophysiology, neuroscience, electrophysiology. 